\title{
AN ALTERNATIVE PROOF OF THE LINEARITY OF THE SIZE-RAMSEY NUMBER OF PATHS
}

\author{
ANDRZEJ DUDEK AND PAWEŁ PRAŁAT
}

\begin{abstract}
The size-Ramsey number $\hat{r}(F)$ of a graph $F$ is the smallest integer $m$ such that there exists a graph $G$ on $m$ edges with the property that every colouring of the edges of $G$ with two colours yields a monochromatic copy of $F$. In 1983, Beck provided a beautiful argument that shows that $\hat{r}\left(P_{n}\right)$ is linear, solving a problem of Erdős. In this note, we provide another proof of this fact that actually gives a better bound, namely, $\hat{r}\left(P_{n}\right)<137 n$ for $n$ sufficiently large.
\end{abstract}

\section{Introduction}

For given two finite graphs $F$ and $G$, we write $G \rightarrow F$ if for every colouring of the edges of $G$ with two colours (say blue and red) we obtain a monochromatic copy of $F$ (that is, a copy that is either blue or red). The size-Ramsey number of a graph $F$, introduced by Erdős, Faudree, Rousseau and Schelp [7] in 1978, is defined as follows:

$$
\hat{r}(F)=\min \{|E(G)|: G \rightarrow F\} .
$$

In this note, we consider the size-Ramsey number of the path $P_{n}$ on $n$ vertices. It is obvious that $\hat{r}\left(P_{n}\right)=\Omega(n)$ and that $\hat{r}\left(P_{n}\right)=O\left(n^{2}\right)$ (for example, $K_{2 n} \rightarrow P_{n}$ ) but the exact behaviour of $\hat{r}\left(P_{n}\right)$ was not known for a long time. In fact, Erdős [6] offered $\$ 100$ for a proof or disproof that

$$
\hat{r}\left(P_{n}\right) / n \rightarrow \infty \quad \text { and } \quad \hat{r}\left(P_{n}\right) / n^{2} \rightarrow 0 .
$$

The problem was solved by Beck [2] in 1983 who, quite surprisingly, showed that $\hat{r}\left(P_{n}\right)<$ $900 n$ for sufficiently large $n$. A variant of his proof was provided by Bollobás [5] and it gives $\hat{r}\left(P_{n}\right)<720 n$ for sufficiently large $n$. It is worth mentioning that both of these bounds are not explicit constructions. Later Alon and Chung [1] gave an explicit construction of graphs $G$ on $O(n)$ vertices with $G \rightarrow P_{n}$.

Here we provide an alternative and elementary proof of the linearity of the size-Ramsey number of paths that gives a better bound. The proof relies on a simple observation, Lemma 2.1, which may be applicable elsewhere.

Theorem 1.1. For $n$ sufficiently large, $\hat{r}\left(P_{n}\right)<137 n$.

In order to show the result, similarly to Beck and Bollobás, we are going to use binomial random graphs. The binomial random graph $G(n, p)$ is the random graph $G$ on vertex set $[n]$ for which for every pair $\{i, j\} \in\left(\begin{array}{c}{[n]} \\ 2\end{array}\right),\{i, j\}$ appears independently as an edge in $G$

Key words and phrases. Ramsey theory, size Ramsey number, random graphs.

The first author is supported in part by Simons Foundation Grant \#244712 and by a grant from the Faculty Research and Creative Activities Award (FRACAA), Western Michigan University.

The second author is supported in part by NSERC and Ryerson University.

Work done during a visit to the Institut Mittag-Leffler (Djursholm, Sweden). 
with probability $p$. Note that $p=p(n)$ may, and usually does, tend to zero as $n$ tends to infinity. All asymptotics throughout are as $n \rightarrow \infty$. We say that a sequence of events $\mathcal{E}_{n}$ in a probability space holds asymptotically almost surely (or a.a.s.) if the probability that $\mathcal{E}_{n}$ holds tends to 1 as $n$ goes to infinity. For simplicity, we do not round numbers that are supposed to be integers either up or down; this is justified since these rounding errors are negligible to the asymptomatic calculations we will make.

\section{Proof of Theorem 1.1}

We start with the following elementary observation.1

Lemma 2.1. Let $c>1$ be a real number and let $G=(V, E)$ be a graph on cn vertices. Suppose that the edges of $G$ are coloured with the colours blue and red and there is no monochromatic $P_{n}$. Then the following two properties hold:

(i) there exist two disjoint sets $U, W \subseteq V$ of size $n(c-1) / 2$ such that there is no blue edge between $U$ and $W$,

(ii) there exist two disjoint sets $U^{\prime}, W^{\prime} \subseteq V$ of size $n(c-1) / 2$ such that there is no red edge between $U^{\prime}$ and $W^{\prime}$.

Proof. We perform the following algorithm on $G$ and construct a blue path $P$. Let $v_{1}$ be an arbitrary vertex of $G$, let $P=\left(v_{1}\right), U=V \backslash\left\{v_{1}\right\}$, and $W=\emptyset$. We investigate all edges from $v_{1}$ to $U$ searching for a blue edge. If such an edge is found (say from $v_{1}$ to $v_{2}$ ), we extend the blue path as $P=\left(v_{1}, v_{2}\right)$ and remove $v_{2}$ from $U$. We continue extending the blue path $P$ this way for as long as possible. Since there is no monochromatic $P_{n}$, we must reach the point of the process in which $P$ cannot be extended, that is, there is a blue path from $v_{1}$ to $v_{k}(k<n)$ and there is no blue edge from $v_{k}$ to $U$. This time, $v_{k}$ is moved to $W$ and we try to continue extending the path from $v_{k-1}$, reaching another critical point in which another vertex will be moved to $W$, etc. If $P$ is reduced to a single vertex $v_{1}$ and no blue edge to $U$ is found, we move $v_{1}$ to $W$ and simply re-start the process from another vertex from $U$, again arbitrarily chosen.

An obvious but important observation is that during this algorithm there is never a blue edge between $U$ and $W$. Moreover, in each step of the process, the size of $U$ decreases by 1 or the size of $W$ increases by 1 . Finally, since there is no monochromatic $P_{n}$, the number of vertices of the blue path $P$ is always smaller than $n$. Hence, at some point of the process both $U$ and $W$ must have size at least $n(c-1) / 2$. Part (i) now holds after removing some vertices from $U$ or $W$, if needed, so that both sets have sizes precisely $n(c-1) / 2$.

Part (ii) can be proved by a symmetric argument; this time the algorithm tries to build a red path. The proof is finished.

Now, we prove the following straightforward properties of random graphs. For every two disjoint sets $S$ and $T, e(S, T)$ denotes the number of edges between $S$ and $T$.

Lemma 2.2. Let $c=7.29$ and $d=5.14$, and consider $G=(V, E) \in G(c n, d / n)$. Then, the following two properties hold a.a.s.:

(i) $|E(G)|=(1+o(1)) n c^{2} d / 2<137 n$,

(ii) for every two disjoint sets of vertices $S$ and $T$ such that $|S|=|T|=n(c-3) / 4$ we have $e(S, T) \neq 0$.

\footnotetext{
${ }^{1} \mathrm{~A}$ similar result was independently obtained by Pokrovskiy [10].
} 
Proof. Part (i) is obvious. The expected number of edges in $G$ is $\left(\begin{array}{c}c n \\ 2\end{array}\right) \frac{d}{n}=(1+o(1)) n c^{2} d / 2$, and the concentration around the expectation follows immediately from Chernoff's bound.

For part (ii), let $X$ be the number of pairs of disjoint sets $S$ and $T$ of desired size such that $e(S, T)=0$. Putting $\alpha=\alpha(c)=(c-3) / 4$ for simplicity, we get

$$
\begin{aligned}
\mathbb{E}[X] & =\left(\begin{array}{c}
c n \\
\alpha n
\end{array}\right)\left(\begin{array}{c}
(c-\alpha) n \\
\alpha n
\end{array}\right)\left(1-\frac{d}{n}\right)^{\alpha n \cdot \alpha n} \\
& \leq \frac{(c n) !}{(\alpha n) !(\alpha n) !((c-2 \alpha) n) !} \exp \left(-d \alpha^{2} n\right) .
\end{aligned}
$$

Using Stirling's formula $\left(x !=(1+o(1)) \sqrt{2 \pi x}(x / e)^{x}\right)$ we get that $\mathbb{E}[X] \leq \exp (f(c, d) n)$, where

$$
f(c, d)=c \ln c-2 \alpha \ln \alpha-(c-2 \alpha) \ln (c-2 \alpha)-d \alpha^{2} .
$$

Putting numerical values of $c$ and $d$ into the formula, we get $f(c, d)<-0.008$ and so $\mathbb{E}[X] \rightarrow 0$ as $n \rightarrow \infty$. (The values of $c$ and $d$ were chosen so as to minimize $c^{2} d / 2$ under the condition $f(c, d)<0$.) Now part (ii) holds by Markov's inequality.

Now, we are ready to prove the main result.

Proof of Theorem 1.1. Let $c=7.29$ and $d=5.14$, and consider $G=(V, E) \in G(c n, d / n)$. We show that a.a.s. $G \rightarrow P_{n}$ which will finish the proof by Lemma 2.2(i).

For a contradiction, suppose that $G \nrightarrow \rightarrow P_{n}$. Thus, there is a blue-red colouring of $E$ with no monochromatic $P_{n}$. It follows (deterministically) from Lemma 2.1(i) that $V$ can be partitioned into three sets $P, U, W$ such that $|P|=n,|U|=|W|=n(c-1) / 2$, and there is no blue edge between $U$ and $W$. Similarly, by Lemma 2.1(ii), $V$ can be partitioned into three sets $P^{\prime}, U^{\prime}, W^{\prime}$ such that $\left|P^{\prime}\right|=n,\left|U^{\prime}\right|=\left|W^{\prime}\right|=n(c-1) / 2$, and there is no red edge between $U^{\prime}$ and $W^{\prime}$.

Now, consider $X=U \cap U^{\prime}, Y=U \cap W^{\prime}, X^{\prime}=W \cap U^{\prime}, Y^{\prime}=W \cap W^{\prime}$ and let $x=|X|, y=$ $|Y|, x^{\prime}=\left|X^{\prime}\right|, y^{\prime}=\left|Y^{\prime}\right|$ be their sizes, respectively. Observe that

$$
x+y=\left|U \cap\left(U^{\prime} \cup W^{\prime}\right)\right|=\left|U \backslash P^{\prime}\right| \geq|U|-\left|P^{\prime}\right|=n(c-3) / 2 .
$$

Similarly, one can show that $x^{\prime}+y^{\prime} \geq n(c-3) / 2, x+x^{\prime} \geq n(c-3) / 2$, and that $y+y^{\prime} \geq$ $n(c-3) / 2$. We say that a set is large if its size is at least $n(c-3) / 4$; otherwise, we say that it is small. We need the following straightforward observation.

Claim. Either both $X$ and $Y^{\prime}$ are large or both $Y$ and $X^{\prime}$ are large.

(In fact one can easily show that the constant $(c-3) / 4$ in the definition of being large is optimal.)

Proof of the claim. For a contradiction, suppose that at least one of $X, Y^{\prime}$ is small and at least one of $Y, X^{\prime}$ is small, say, $X$ and $Y$ are small. But this implies that $x+y<$ $n(c-3) / 4+n(c-3) / 4=n(c-3) / 2$, which contradicts (1). The remaining three cases are symmetric, and so the claim holds.

Now, let us come back to the proof. Without loss of generality, we may assume that $X=U \cap U^{\prime}$ and $Y^{\prime}=W \cap W^{\prime}$ are large. Since $X \subseteq U$ and $Y^{\prime} \subseteq W$, there is no blue edge between $X$ and $Y^{\prime}$. Similarly, one can argue that there is no red edge between $X$ and $Y^{\prime}$, and so $e\left(X, Y^{\prime}\right)=0$. On the other hand, Lemma 2.2(ii) implies that a.a.s. 
$e\left(X, Y^{\prime}\right) \neq 0$, reaching the desired contradiction. It follows that a.a.s. $G \rightarrow P_{n}$ which finishes the proof.

\section{REMARKS}

In this note we showed that $\hat{r}\left(P_{n}\right)<137 n$. On the other hand, the best known lower bound, $\hat{r}\left(P_{n}\right) \geq(1+\sqrt{2}) n-2$, was given by Bollobás [4] who improved the previous result of Beck [3] that shows that $\hat{r}\left(P_{n}\right) \geq \frac{9}{4} n$. Decreasing the gap between the lower and upper bounds might be of some interest. One approach to improving the upper bound could be to deal with non-symmetric cases in our claim or to use random $d$-regular graphs instead of binomial graphs.

Another related problem deals with longest monochromatic paths in $G(n, p)$. Observe that it follows from the proof of Theorem 1.1 that for every $\omega=\omega(n)$ tending to infinity arbitrarily slowly together with $n$ we have that a.a.s. any 2-colouring of the edges of $G(n, \omega / n)$ yields a monochromatic path of length $\frac{(1-\varepsilon)}{3} n$ for an arbitrarily small $\varepsilon>0$. On the other hand, a simple construction of Gerencsér and Gyárfás [8] shows that such path cannot be longer than $\frac{2}{3} n$. We conjecture that actually $(1+o(1)) \frac{2}{3} n$ is the right answer for random graphs with average degree tending to infinity 2

\section{ACKNOWLEDGMENT}

We would like to thank to the referees and editors for their valuable comments and suggestions.

\section{REFERENCES}

[1] N. Alon and F.R.K. Chung, Explicit construction of linear sized tolerant networks, Discrete Math. 72 (1988), 15-19.

[2] J. Beck, On size Ramsey number of paths, trees, and circuits I, J. Graph Theory 7 (1983), 115-129.

[3] J. Beck, On size Ramsey number of paths, trees and circuits II, Mathematics of Ramsey theory, 34-45, Algorithms Combin. 5, Springer, Berlin, 1990.

[4] B. Bollobás, Extremal graph theory with emphasis on probabilistic methods, CBMS Regional Conf. Ser. in Math. 62 (1986), viii+64 pp.

[5] B. Bollobás, Random Graphs, Cambridge University Press, Cambridge, 2001.

[6] P. Erdős, On the combinatorial problems which I would most like to see solved, Combinatorica 1 (1981), no. 1, 25-42.

[7] P. Erdős, R. Faudree, C. Rousseau, and R. Schelp, The size Ramsey number, Period. Math. Hungar. 9 (1978), no. 1-2, 145-161.

[8] L. Gerencsér and A. Gyárfás, On Ramsey-type problems, Ann. Univ. Sci. Budapest. Eötvös Sect. Math. 10 (1967), 167-170.

[9] S. Letzter, Path Ramsey number for random graphs, ArXiv e-prints, (2014).

[10] A. Pokrovskiy, Partitioning edge-coloured complete graphs into monochromatic cycles and paths, J. Combin. Theory Ser. B 106 (2014), 70-97.

Department of Mathematics, Western Michigan University, Kalamazoo, Mi, USA

E-mail address: andrzej.dudek@wmich.edu

Department of Mathematics, Ryerson University, Toronto, On, Canada

E-mail address: pralat@ryerson.ca

${ }^{2}$ The conjecture was recently proved by Letzter $[9]$. 